\title{
Effects of local vibrations on skeletal muscle trophism in elderly people: Mechanical, cellular, and molecular events
}

\author{
TIZIANA PIETRANGELO ${ }^{1}$, ROSA MANCINELLI ${ }^{1}$, LUANA TONIOLO $^{2}$, LINA CANCELLARA $^{2}$, \\ ANTONIO PAOLI $^{2}$, CRISTINA PUGLIELLI ${ }^{1}$, PIERPAOLO IODICE ${ }^{1}$, CHRISTIAN DORIA $^{1}$, \\ GERARDO BOSCO $^{1}$, LUIGI D'AMELIO ${ }^{1}$, GUGLIELMO DI TANO ${ }^{1}$, STEFANIA FULLE ${ }^{1}$, \\ RAOUL SAGGINI $^{1}$, GIORGIO FANO ${ }^{1}$ and CARLO REGGIANI ${ }^{2}$ \\ ${ }^{1}$ Dipartimento Scienze Mediche di Base ed Applicate, Istituto Interuniversitario di Miologia, Centro di \\ Studi sull'Invecchiamento, University G. d'Annunzio Chieti-Pescara, via dei Vestini 29, I-66013 Chieti; \\ ${ }^{2}$ Dipartimento Anatomia e Fisiologia, Università di Padova, via Marzolo 3, I-35131 Padova, Italy
}

Received February 23, 2009; Accepted May 7, 2009

DOI: 10.3892/ijmm_00000259

\begin{abstract}
Several studies have examined the effects of vibrations on muscle mass and performance in young healthy people. We studied the effects of vibrations on muscles of elderly male and female volunteers (65-85 years of age) diagnosed with sarcopenia. We applied mechanical vibrations locally (local vibrational training) to the thigh muscles at $300 \mathrm{~Hz}$ for a period of 12 weeks, starting with a session of 15 min stimulation once a week and increasing to three sessions of 15 min per week. Treated muscles displayed enhanced maximal isometric strength and increased content of fast MyHC-2X myosin. Single muscle fiber analysis did not show any change in cross-sectional area or in specific tension. Analysis of transcriptional profiles by microarray revealed changes in gene expression after 12 weeks of local vibrational training. In particular, pathways related with energy metabolism, sarcomeric protein balance and oxidative stress response were affected. We conclude that vibration treatment is effective in counteracting the loss of muscular strength associated with sarcopenia and the mode of action of vibration is based on cellular and molecular changes which do not include increase in fiber or muscle size.
\end{abstract}

\section{Introduction}

Aging is associated with progressive loss of neuromuscular function. The term sarcopenia is commonly used to describe the loss of skeletal muscle mass and strength that occurs in connection with biological aging in the elderly. The onset of sarcopenia is generally assumed to occur around 60 years of

Correspondence to: Dr Tiziana Pietrangelo, Dipartimento Scienze Mediche di Base ed Applicate, University 'G. d'Annunzio' Chieti-Pescara, Via dei Vestini 29, I-66013 Chieti, Italy

E-mail: tiziana@unich.it

Key words: vibrations, skeletal muscle, single fibers, gene expression age, with atrophy being an important symptom. The progression of sarcopenia is influenced by several factors, including genetic components, lifestyle, age-related diseases, decreases in the levels of hormones (GH, testosterone, IGF-1), loss of motor units, and decreased regenerative capacity of skeletal muscle stem cells $(1,2)$. Atrophic conditions in the elderly are exacerbated when diseases force them to bed. The main countermeasure is regular and moderate exercise, but, besides general advice to stay active, there are no definitive indications for optimal training and/or treatment of sarcopenia $(3,4)$.

In recent years, mechanical muscle vibration received considerable attention as a useful method of muscle stimulation in clinical therapy and sports training, but the results remain controversial. Only a few studies described specific vibrational training protocols, and this lack of information generates uncertainties regarding the most effective vibration intensities, frequencies, and application protocols. When frequency is considered, the main question is whether vibrations are applied to the whole body or to specific muscles. The body tolerates a vibration frequency in the range $20-50 \mathrm{~Hz}$, whereas the level for local application to specific skeletal muscles is in the range of $300-500 \mathrm{~Hz}$.

In view of the diversity of applied treatments, a precise comparison of results reported in scientific literature is difficult, and for this reason we report data on different vibrational protocols without extensive discussion. For instance, Bosco and coworkers showed positive effects of passive whole-body vibration under different conditions, reporting significant increases in muscle strength and power (5), elevations in plasma concentrations of testosterone and growth hormone $(6,7)$, and improvement in neuromuscular properties (8). Another study showed that maximal isometric voluntary contraction increased (in young men) only when vibration was combined with squat training (7). De Ruiter and colleagues studied the effects on young men over a period of 11 weeks of whole-body vibration at $30 \mathrm{~Hz}$ and found that neither the strength nor the contractile properties of the knee extensor muscle improved (9). It is also worth remembering that negative effects of whole-body vibration on health were reported. Hand-arm vibration syndrome and 
Table I. Anthropometric parameters of study participants.

\begin{tabular}{lcccccc}
\hline & $\begin{array}{c}\text { Age } \\
\text { years }\end{array}$ & $\begin{array}{c}\text { Height } \\
\mathrm{cm}\end{array}$ & $\begin{array}{c}\text { Weight pre-training } \\
\mathrm{kg}\end{array}$ & $\begin{array}{c}\text { Weight post-training } \\
\mathrm{kg}\end{array}$ & $\begin{array}{c}\text { BMI pre-training } \\
\mathrm{kgm}^{-2}\end{array}$ & $\begin{array}{c}\text { BMI post-training } \\
\mathrm{kgm}^{-2}\end{array}$ \\
\hline Male & $75.3 \pm 6.9$ & $163.0 \pm 5.0$ & $76.9 \pm 6.7$ & $75.3 \pm 6.2$ & $29.1 \pm 3.5$ & $29.0 \pm 3.3$ \\
Female & $71.0 \pm 5.7$ & $159.0 \pm 5.0$ & $71.6 \pm 18.8$ & $66.4 \pm 15$ & $28.3 \pm 8.1$ & $28.2 \pm 7.6$ \\
\hline
\end{tabular}

Data reported in the Table are the anthropometric measurements of male and female elderly volunteers. The values are means \pm SD.

vascular disorders $(10,11)$, low back pain (12) and spinal health risks (13), were reported in vibration-exposed workers.

The possible involvement of the nervous system in modifications of muscle performance induced by vibration has been the focus of several studies (14). It was hypothesized that mechanical stimuli are transmitted to sensory receptors of muscles, most likely the spindles, and that receptor activation results in the reflex stimulation of motor units, as seen in the tonic vibration reflex (15). However, the tonic vibration stretch reflex was originally described as the result of a brief exposure to high-frequency stimulation applied directly to a tendon $(16,17)$. Any activation prolonged for tens of seconds could induce a reduction in muscle spindle firing frequency and, as a consequence, a decrease in muscle activation (15). Moreover, muscle spindle firing induced by vibration excites not only motor neurons but also interneurons in the spinal cord, which reciprocally inhibit the motor neurons of antagonist muscles (18). Brunetti and coworkers studied posture stability after vibratory stimulation following anterior cruciate ligament reconstruction. These authors concluded that vibration leads to a faster and more complete recovery of equilibrium, confirming a role for vibration in proprioceptive stimulation (19). In conclusion, it remains difficult to find a rationale that is logically used to design vibration treatment and to predict treatment outcomes.

In this study, we aimed to determine whether a training program of passive muscle stimulation, in which local mechanical vibrations at high frequency $(300 \mathrm{~Hz})$ were applied to the lower limbs in the absence of any voluntary muscle contraction, induces an increase in muscle mass and strength in elderly subjects showing signs of sarcopenia. Isometric strength developed in maximal voluntary contractions by knee extensor muscles, and thigh circumference, were the parameters selected to evaluate the impact of local muscle mechanical vibrations in elderly male and female volunteers, and thigh circumference was used as an indicator of structural modifications. Additionally, using small fragments of tissue from needle biopsies of the vastus lateralis $(V L)$ muscle (preand post-training), some cellular features (fiber types, fiber cross-sectional area, single fiber tension development) and gene expression profiles were analyzed.

\section{Materials and methods}

Subjects. The study involved nine elderly people (four males and five females) with diagnoses of sarcopenia according to the criteria of the Centers for Disease Control and Prevention (CDCP). Anthropometric characteristics are summarized in
Table I. The study was approved by the local ethics committee, and was performed in accordance with the 1964 Declaration of Helsinki. All individuals provided written informed consent before participating in the study.

The inclusion criteria were as follows: diagnosis of sarcopenia; normal ECG and blood pressure; and absence of bone/joint, metabolic, or cardiovascular diseases. Exclusion criteria were the presence of metabolic and/or cardiovascular diseases, evidence of hereditary or acquired muscular disease, or diagnosis of respiratory or psychiatric disorders. No subject was under treatment with testosterone or other pharmacological interventions known to influence muscle mass.

Training protocol and experimental design. The conditioning protocol consisted of application of local high-intensity vibrations on the lower limbs using the VISS apparatus. The VISS device (Vissman, Rome, Italy) is a tool capable of producing acoustic waves of different frequencies without affecting the set width. The device is not an acoustic wave generator, but rather a flux modulator, and has two components. These are a compressor delivering pressure in the range 0-400 millibar and a modulator producing an oscillatory air flux to create acoustic waves through a two-way rotating valve. The transducer develops a time-modulated sinusoidal wave $(300 \mathrm{~Hz})$. During application of vibration, subjects were invited to avoid isometric contractions of the treated muscle. The experimental protocol required that local mechanoacoustic vibratory stimulation was applied on the skin of the distal part of the quadriceps close to the tendon insertions of the intermedius femoris, rectus femoris, vastus medialis, and vastus lateralis muscles. The entire treatment lasted 12 weeks. The duration of each application was $15 \mathrm{~min}$ and the frequency chosen was $300 \mathrm{~Hz}$. From weeks 1 to 8 the subjects received one application per week, whereas from weeks 9 to 12 they received three applications per week. We refer to this type of stimulation protocol as 'local high-intensity vibrational training'.

Two weeks before the training period, enrolled subjects were familiarized with the test session protocol. Isometric tests were performed a week before (pre-session) and a week after the conditioning period (post-session), to measure the bilateral maximal isometric strength of the lower limbs, the body mass index (BMI), and thigh circumference, assessed with a measuring tape at two levels on the dominant leg of each standing subject. The circumferences measured were the maximal circumference at one-third of the distance between the trochanter and the tibial-femoral joint space, and the minimum circumference above the knee. Skinfold measurements were next recorded at the anterior mid-thigh using a skinfold caliper. 
Sixteen weeks after the end of the conditioning period of local high-intensity vibrational training, the isometric test was repeated to monitor lower limb strength level.

Isometric strength measurement. Bilateral isometric torque developed by the knee extensor muscles was measured during maximal voluntary contractions using a Leg Extension machine (Panatta Sport; Apiro [MC], Italy) equipped with a load cell (Globus Italia, Codognè [TV], Italy). Participants were seated with the trunk-thigh and the knee joint angles at $90^{\circ}$. Subjects performed maximal voluntary isometric contractions of the knee extensors three times. Isometric contractions lasted for $5 \mathrm{sec}$, and were separated by $2 \mathrm{~min}$ rest intervals. The highest value of torque attained was taken as the isometric contraction strength. In each subject, the variation of knee extension isometric force was expressed as a percentage of the pretraining value.

Cellular and molecular analysis of muscle biopsies. Muscle biopsies were obtained using a semi-automatic needle (Precisa 13 Gauge; Hospital Service, Rome, Italy) from the $V L$ muscle at a level corresponding to one-third of the distance from the upper margin of the patella to the anterior superior iliac spine, after local anesthesia with lidocaine $(0.5 \%, \mathrm{w} / \mathrm{v})$. In each subject, several samples were collected from the same needle insertion and were divided into three groups: (i) samples immersed in skinning solution (see below) were used for dissection of single muscle fibers; (ii) samples immersed in Laemmli buffer (see below) were used for gel electrophoresis; and, (iii) samples immersed in Trizol reagent (Invitrogen, Paisley, UK) were used for extraction of RNA for analysis of transcriptional profiles.

Muscle fibers, mechanical characterization. Muscle biopsy fragments for single fiber dissection were immersed in icecold skinning solution with $50 \%(\mathrm{v} / \mathrm{v})$ glycerol. Skinning solution is a high-potassium, high-EGTA solution which depolarizes membranes, removes calcium, and induces a rigor state, thus ensuring optimal conditions for fiber preservation. The fragments were stored at $-20^{\circ} \mathrm{C}$ and analyzed within 2 weeks of sampling. On the day of the experiment, the skinning-glycerol mixture was washed off and replaced with ice-cold skinning solution containing ATP, to induce fiber relaxation. Single fibers were manually dissected under a stereo-microscope (x10-60 magnification). Following dissection, fibers were bathed for $30 \mathrm{~min}$ in skinning solution containing $1 \%(\mathrm{v} / \mathrm{v})$ Triton $\mathrm{X}-100$ to ensure complete membrane solubilization. Fiber segments 1-2 mm in length were cut, and light aluminum clips were applied at both ends. Skinning, relaxing, pre-activating, and activating solutions employed for single fiber experiments were prepared as described previously (20). Fiber segments were transferred to the experimental apparatus, and cross-sectional area and tension development during maximal calcium-activated isometric contractions at $12^{\circ} \mathrm{C}$ were measured according to a previously described procedure (21).

Electrophoretic separation and quantification of myosin heavy chain (MyHC) isoforms. MyHC isoform composition was determined in biopsy samples. Muscle biopsy fragments were stored in Laemmli solution (Tris $62.5 \mathrm{mM}$, Glycerol $10 \%$ [v/v], SDS $2.3 \%$ [w/v], B-mercaptoethanol 5\% [v/v], with
E-64 0.1\% [w/v] and leupeptin $0.1 \%$ [w/v] as anti-proteolytic factors; $\mathrm{pH}$ 6.8). After heating for $5 \mathrm{~min}$ at $80^{\circ} \mathrm{C}$ appropriate amounts of the protein suspension were loaded onto polyacrylamide gels $(\sim 1 \mu \mathrm{g}$ of total protein/lane). Separation of MyHC isoforms was achieved on $8 \%(\mathrm{w} / \mathrm{v})$ gels $(18 \mathrm{~cm} \mathrm{x}$ $16 \mathrm{~cm} \mathrm{x} 1 \mathrm{~mm}$ ) at $70 \mathrm{~V}$ for $1.5 \mathrm{~h}$ and at $230 \mathrm{~V}$ for a further time according to the guidelines of Talmadge and Roy (22). After silver staining, three separate bands were detected in the 200-kDa region, corresponding to MyHC-1, $-2 \mathrm{~A}$, and $-2 \mathrm{X}$, in order of migration from fastest to slowest. To quantify myosin isoform distribution, densitometric analyses of silverstained bands were performed on at least two independent electrophoretic runs of each biopsy sample fragment. The mean values represent the measurements presented. Gel patterns were digitized with an EPSON 1650 scanner at a resolution of 1,200 dpi. Each band was characterized by a value of the Brightness-Area Product (BAP), using a constant threshold after black/white inversion employing Adobe Photoshop software. From each gel, BAP values for the bands identified as MyHC isoforms were summed and the BAP value for each isoform was expressed as a percentage of the total. The reproducibility of the procedure was confirmed by calculating isoform ratios of selected samples from gels loaded with different amounts of such samples.

Gene expression profile. A high-density oligonucleotide microarray technique was used to identify variations in gene expression induced by mechanical vibration. This technique involves RNA isolation, amplification, and labeling. The human oligonucleotide gene set consisting of 21,329 (70-mer) oligonucleotides (Operon version 2.0), designed on the basis of the Human Unigene clusters, was employed. RNA was extracted from the biopsy samples of three different elderly subjects before training, and at the end of the vibrational training period. Very pure RNA samples from each subject were amplified and labeled. Microarray co-hybridization involved simultaneous hybridization with pre-training and post-training samples and dye-swap duplicated microarrays were analyzed for each subject. Arrays were scanned, and recorded fluorescence intensities were subjected to Lowess normalization (23). The expression of each gene was defined as the $\log$ base- 2 of the ratio between the intensity of cyanine-coupled aaRNA from post-training samples and that of cyanine-coupled aaRNA from pre-training samples $\left(\log _{2}\right.$ $\left.\mathrm{I}_{\text {post-training }} / \mathrm{I}_{\text {pre-training }}\right)$. Differentially expressed genes were selected using a per-mutation test procedure known as the 'Significance Analysis of Microarrays' (SAM), which defines genes with a computed score larger than the threshold value as showing significant variation. The false discovery rate (FDR) associated with the given threshold was additionally calculated from permutation data.

Statistical analysis. Data were expressed as means \pm SE (Figs.) or SD (Tables). Statistical significance was set at $\mathrm{p}<0.05$ and was calculated using the unpaired Student's t-test, with Welch's correction. Prism5 GraphPad software (Abacus Concepts GraphPad Software, San Diego, CA) was employed for statistical analyses. Gene expression analysis was based on SAM data and variations are expressed as means with SD. 



Figure 1. Variations in bilateral isometric strength of leg extensor muscles treated with local muscle vibration at $300 \mathrm{~Hz}$ in nine elderly male (A) and female (B) subjects. The histograms show the percentage increases in bilateral isometric strength, based on leg extension, at different times during the period of stimulation. 0 week, pre-training; 4,8 , and 12 weeks, intervals during the stimulation period when strength was measured. During the first 8 weeks, treatment was based on a single vibration application $(300 \mathrm{~Hz})$ of 15 min per week. From weeks 8 to 12 , treatment was increased to three applications of $15 \mathrm{~min}$ per week $(300 \mathrm{~Hz})$. Treatment was interrupted after 12 weeks, and strength was measured again at week 28 . Isometric strength measurements from the pre-training sessions are taken as $100 \%$. Significant variations, ${ }^{*} \mathrm{p}<0.05,{ }^{* *} \mathrm{p}<0.01$

\section{Results}

Maximal isometric strength of knee extensor muscles. Isometric strength of leg extensor muscles was measured before, and after 4,8 , and 12 weeks of vibrational training. The application of local vibration to the lower part of the thighs improved isometric strength (Fig. 1). In both male and female subjects (anthropometric characteristic in Table I), the increase was appreciable, beginning at 4 weeks (females) and 8 weeks (males) of vibration training. Importantly, during the first 8 weeks of the procedure, stimulation was scheduled only once a week. This increase in strength remained at a plateau up to week 12 , even though training frequency was increased to three times per week from week 8 (see Training protocol). In general, the response was better in female than in male subjects. In male subjects, isometric strength increased by $\sim 25 \%$ at weeks 4 and 8 , with a significant increase at week 12 of training $(141.7 \pm 12.7 \%, \mathrm{n}=4, \mathrm{p}<0.05)$. Follow-up measurements after 28 weeks revealed that the increase in


In female subjects, the increase in bilateral isometric limb strength was higher than in males, commencing at week 4 (148.5 $\pm 6.5 \%, \mathrm{n}=5, \mathrm{p} \leq 0.001)$, and remained significant until the end of the training period $(181.2 \pm 19.3 \%, \mathrm{n}=4, \mathrm{p} \leq 0.001)$. The increase in strength at 12 weeks of stimulation was not significantly different between female and male subjects.

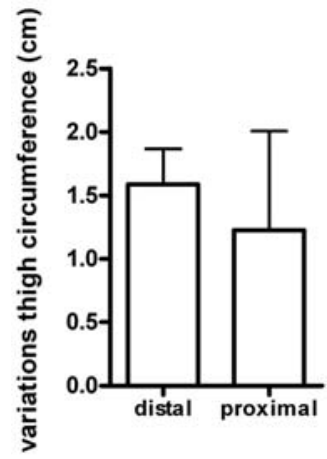

Figure 2. Variations in thigh circumference. Two thigh circumferences, one distal (just above the knee) and one proximal (at 2/3 of the knee-trochanter distance, to avoid the adductor muscles) were measured before the beginning of treatment (pre-training) and after 12 weeks of treatment (posttraining). Changes are expressed in $\mathrm{cm}$. As average, the distal and proximal circumferences showed small and insignificant increases after vibrational training in both male and female subjects.

Variations of leg extensor muscle mass were evaluated from the measurements of two thigh circumferences, one distal (just above the knee), and one proximal (at 2/3 of the kneetrochanter distance), to avoid the influence of adductor muscles. After vibrational training the distal circumferences showed small and insignificant increases $(0.5-3 \mathrm{~cm})$ in both male and female subjects, whereas, with proximal circumferences, variations ranged from a slight decrease in two female subjects to an increase (0.5-6 cm) in other subjects (Fig. 2). Moreover, skinfold measurements at the end of the training period were not significantly different from those measured before training (data not shown). In conclusion, no significant variations in muscle mass accompanied increases in muscle strength.

Muscle fiber cross-sectional area and specific tension. Single muscle fibers were isolated from biopsy fragments of the $V L$ muscle obtained before and after vibrational training. From each biopsy, 8-9 fibers were analyzed, to yield data on a total of 80 fibers both before and after training. The average crosssectional area (CSA) was $3667.0 \pm 310.7 \mu \mathrm{m}^{2}$ in pre-training samples, and $4238.0 \pm 357.4 \mu \mathrm{m}^{2}$ in post-training samples, thus, vibrational training did not significantly modify the CSA of single fibers (Fig. 3A). In specific tension measurements, the isometric strength per unit of fiber area was $177.0 \pm 14.6 \mathrm{mN}$ $\mathrm{mm}^{2}$ in pre-training samples, and $164.0 \pm 17.4 \mathrm{mN} \mathrm{mm}^{-2}$ in post-training specimens. These values were not significantly different (Fig. 3B).

Myosin isoform composition of biopsy samples. The fiber type composition of the $V L$ muscle was determined by analyzing the proportion of slow (MyHC-1) and fast (MyHC-2A and $-2 \mathrm{X}$ ) myosin heavy chain isoforms in biopsy samples taken before and after vibrational training. Myosin isoforms can be considered as molecular markers of fiber types (24). The average values for myosin isoform distribution in males (Fig. 4A) and females (Fig. 4B) are shown. In both groups, fast MyHC-2X levels were increased after vibration training, whereas slow MyHC-1 proportions were significantly lower in post-training compared to pre-training samples. The shift in myosin isoform expression might indicate either fiber 

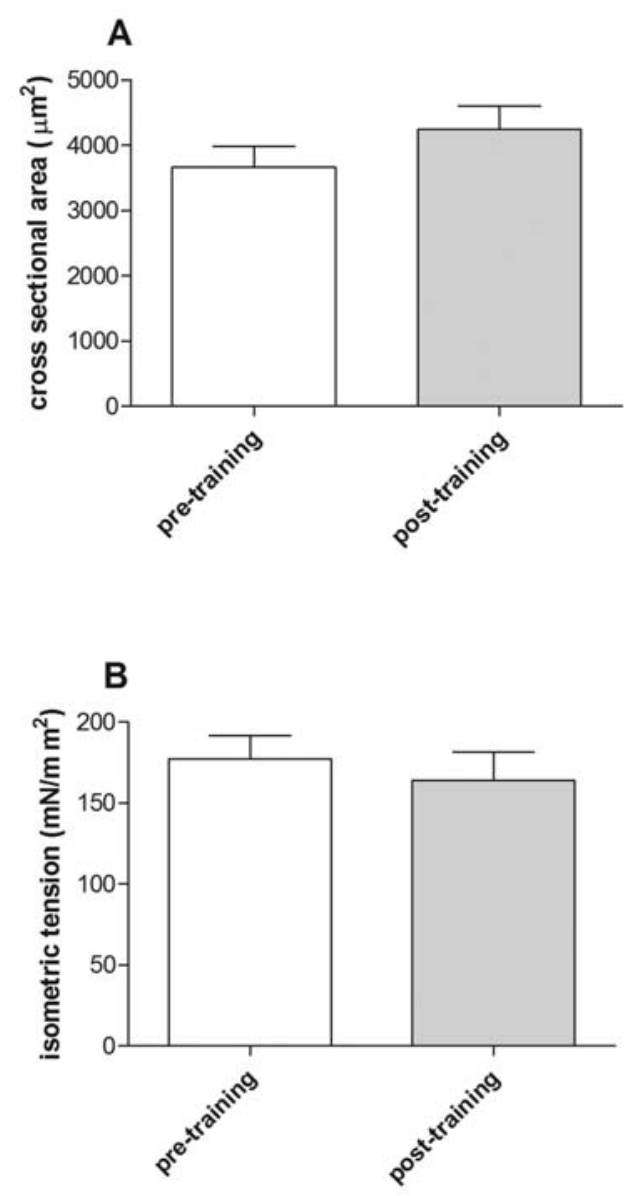

Figure 3. Single-fiber analysis, cross sectional area (A) and specific isometric tension (B). (A) Cross-sectional area (CSA) of single muscle fibers isolated from the biopsy samples from the vastus lateralis muscle before and after vibrational training of 12 weeks. (B) Measurements of specific isometric tension (force/CSA) developed in maximal calciumactivated contraction by the same fibers. In both cases, no significant difference was detected between pre- and post-training samples. For each measurement, a total of 80 fibers were analyzed.

transition (from slow to fast; $2 \mathrm{X}$ ) or differential growth (a specific increase in the size of $2 X$ fibers). The lack of any significant increase in fiber thickness after vibrational training (see Fig. 3) suggests that the change in myosin isoform distribution are caused by a fiber-type transition.

Gene expression profiles. Gene expression profiles from oligonucleotide microarrays were obtained from biopsy samples of three subjects before and after vibrational training. We listed the genes found significantly regulated after the vibrational training on each subject and we assessed possible changes in gene transcription analyzing these regulated genes. Genes that appeared to be significantly affected were further evaluated to elucidate the mechanisms by which vibrationtrained muscles showed performance enhancement. Some genes with well-known muscle activities received particular attention, and were classified according to function. Such categories included, specifically, (i) genes of energy metabolism (Table II); (ii) genes involved in sarcomeric protein synthesis, protein degradation, and calcium homeostasis (Table III); and, (iii) genes dealing with oxidative stress (Table IV).
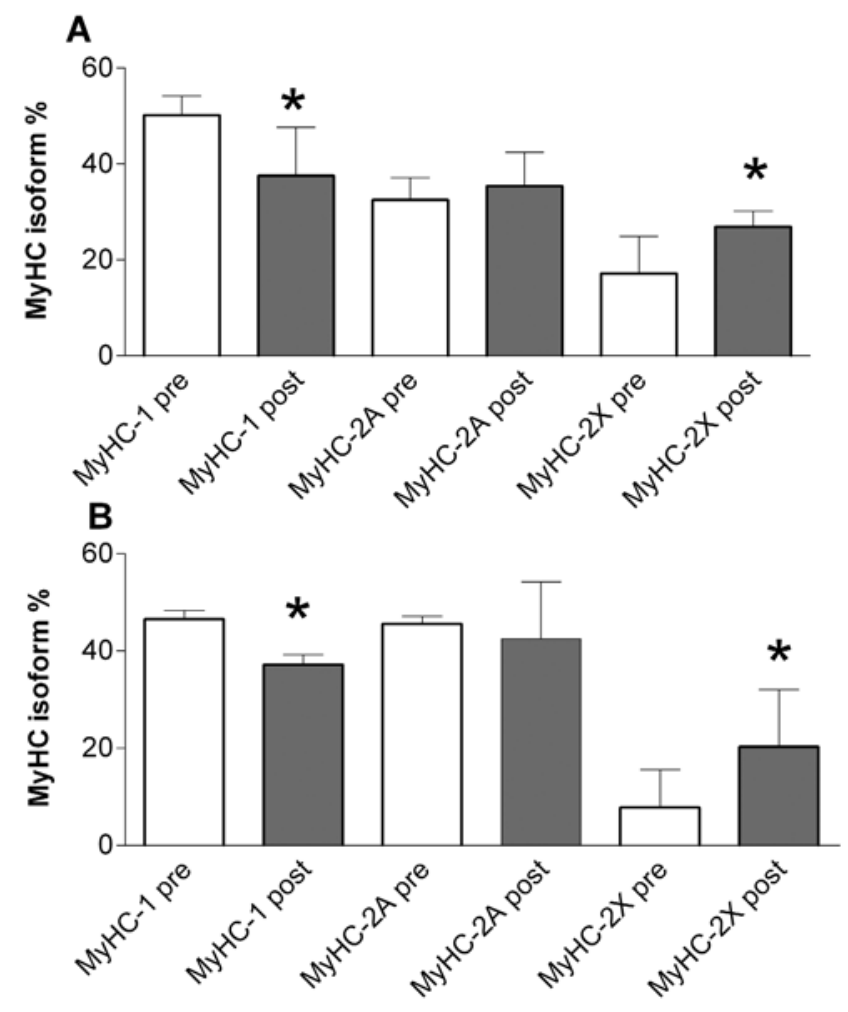

Figure 4. MyHC isoform distribution in biopsy samples collected before and after 12 weeks of vibrational training in nine elderly subjects. MyHC isoform distribution was determined by electrophoretic separation and densitometric analysis of proteins of biopsy samples from the vastus lateralis muscle. A and B show the percentage distributions of $\mathrm{MyHC}$ isoforms (1, $2 \mathrm{~A}$, and $2 \mathrm{X}$ ) in male and female subjects, respectively. The grey columns represent the percentages of the MyHC isoform distribution post-training, and the white columns represent pre-training percentages. (A) The mean percentage of MyHC-1 decreased by $13 \%$, whereas that of MyHC-2X increased by $12 \%$, in elderly male subjects. (B) The mean percentage of MyHC-1 decreased by $11 \%$, whereas that of MyHC-2X increased by $12 \%$, in female subjects. ${ }^{*} \mathrm{p} \leq 0.05$.

Genes involved in energy metabolism. Several enzymes involved in glucose and glycogen metabolism were upregulated after vibration therapy (Table II). We observed increased expression of the phosphoinositide-3-kinase, regulatory subunit, polypeptide 3 (PIK3R3) gene that encodes a binding protein in the insulin-dependent pathway leading to inhibition of glycogen synthase kinase-3 (GSK-3), and that thus mediates net dephosphorylation of glycogen synthase (GS), with concomitant activation of the glycogen pathway (25). It is important to remember that PI3kinase functions upstream to Akt, a signaling factor very relevant to muscle hypertrophy mechanisms (26). Moreover, protein phosphatase regulatory subunits $3 A$ and $3 C$ (PPP1R 3A and 3C), genes encoding key enzymes for glycogen conservation in muscle (25), were upregulated. Another gene showing enhanced expression was dihydrolipoamide dehydrogenase, E3 complex of pyruvate dehydrogenase complex (DLD), a protein of the pyruvate dehydrogenase complex that transforms pyruvate into acetylcoenzyme A. In particular, the E3 component reduces $\mathrm{NAD}^{+}$ to NADH (27). Yet another up-regulated gene of interest was fatty acid CoA ligase that is important for fatty acid activation in the cytoplasm prior to $\beta$-oxidation. Glucan $(1,4-\alpha-)$, branching enzyme 1 (glycogen branching enzyme, [relevant 
Table II. Changes in energy metabolism genes.

\begin{tabular}{|c|c|c|c|c|c|}
\hline Subject & Gene name & $\begin{array}{l}\text { Mean } \\
\log _{2} \mathrm{I}_{\text {post-training }} / I_{\text {pre-training }} \\
\pm \mathrm{SD}\end{array}$ & GB accession & UniGene ID & Gene symbol \\
\hline 1 & $\begin{array}{l}\text { Phosphoinositide-3-kinase, } \\
\text { regulatory subunit, polypeptide } 3(\mathrm{p} 55, \gamma)\end{array}$ & $0.86 \pm 0.39$ & NM_003629 & 88051 & PIK3R3 \\
\hline 2 & $\begin{array}{l}\text { Protein phosphatase } 1 \text {, regulatory (inhibitor) } \\
\text { subunit } 3 \text { A (glycogen and } \\
\text { sarcoplasmic reticulum binding }\end{array}$ & $1.08 \pm 0.13$ & NM_002711 & 127614 & PPP1R3A \\
\hline 2 & $\begin{array}{l}\text { Protein phosphatase 1, regulatory (inhibitor) } \\
\text { subunit 3C }\end{array}$ & $0.99 \pm 0.17$ & ВC012625 & 303090 & PPP1R3C \\
\hline 2 & $\begin{array}{l}\text { Dihydrolipoamide dehydrogenase (E3 component } \\
\text { of pyruvate dehydrogenase complex, } \\
\text { 2-oxo-glutarate complex) }\end{array}$ & $0.85 \pm 0.16$ & NM_000108 & 74635 & DLD \\
\hline 2 & Fatty-acid-coenzyme A ligase, long-chain 2 & $0.91 \pm 0.09$ & NM_021122 & 154890 & FACL2 \\
\hline 1 & $\begin{array}{l}\text { Solute carrier family } 16 \text { (monocarboxylic acid } \\
\text { transporters), member } 4\end{array}$ & $0.84 \pm 0.32$ & NM_004696 & 23590 & SLC16A4 \\
\hline 1 & $\begin{array}{l}\text { Solute carrier family } 21 \text { (organic anion } \\
\text { transporter), member } 11\end{array}$ & $0.65 \pm 0.12$ & NM_013272 & 14805 & SLC21A11 \\
\hline 1 & $\begin{array}{l}\text { Solute carrier family } 6 \text { (neurotransmitter } \\
\text { transporter), member } 14\end{array}$ & $0.80 \pm 0.32$ & NM_007231 & 162211 & SLC6A14 \\
\hline 1 & Ubiquinol-cytochrome c reductase core protein II & $0.69 \pm 0.16$ & NM_003366 & 173554 & UQCRC2 \\
\hline 5 & $\begin{array}{l}\text { Glucan (1,4-alpha-), branching enzyme } 1 \\
\text { (glycogen branching enzyme, } \\
\text { Andersen disease, glycogen storage) }\end{array}$ & $-1.16 \pm 0.15$ & NM_000158 & 1691 & GBE1 \\
\hline
\end{tabular}

The first column specifies in which of the three different elderly subjects (individuals 1, 2, or 5) the change in expression was detected. The second column lists the common names of the different genes up- or down-regulated in the vastus lateralis muscle, when post- and pre-training expression levels were compared. The third column reports changes in expression levels of genes as means of the $\log$ base- 2 of the ratios (log $\mathrm{I}_{\mathrm{post}-\text { training }} / \mathrm{I}_{\text {pre-training }}$ ), with $\mathrm{SD}$. The fourth column lists Gene Bank Accession numbers, the fifth the UniGene Identification numbers, and the sixth specific gene symbols. Genes involved in energy metabolism were classified as upregulated (positive values) or downregulated (negative values).

to Andersen's disease and glycogen storage disease]) (GBE1), which participates in glycogen synthesis, was downregulated.

Expression of specific genes encoding several transporters was enhanced, including genes encoding solute carrier family 16 (monocarboxylic acid transporters), member 4 (SLC16A4) (28), a transport system implicated in both intracellular $\mathrm{pH}$ regulation during muscle contraction and lactate removal. The gene solute carrier family 21 (organic anion transporter), member 11 (SLC21A11), functioning in the transport of organic anions and thyroid hormones, and in modulation of glucose phosphorylation (29), was also upregulated. Moreover, the expression of solute carrier family 6 (neurotransmitter transporter), member 14 (SLC6A14), a gene encoding an amino acid transporter implicated in the utilization of non-glucidic substrates for plastic cellular reorganization and as an energy source (30), was increased. Notably, we observed elevated expression of ubiquinolcytochrome c reductase core protein II (UQCRC2), encoding a cytochrome reductase of the mitochondrial complex III (31).

Genes involved in sarcomeric protein synthesis and degradation, and calcium homeostasis. Several genes encoding sarcomeric proteins were upregulated (Table III). These included actin $\alpha 2$ (ACTA2), one of the six actin isoforms of thin filament proteins, destrin (DSTN), a key enzyme in actin polymerization (32), and myosin, light polypeptide, regulatory, non-sarcomeric (MLCB), together with chaperonin ADP- ribosylation factor-like 5 (ARL5A), and short coiled-coil protein (SCOCO), linked to myosin folding and myofibrillogenesis (33). In the context of protein turnover, we observed upregulation of ubiquitin-specific protease 15 (USP15), involved in the removal of damaged proteins, and downregulation of ubiquitin-conjugating enzyme E2G 1 (UBE2G1), encoding a protease, and an enzyme that conjugates target proteins with ubiquitin, respectively. Moreover, expression of calpain 3 (p94), specifically expressed in skeletal muscle, was enhanced. Calpain 3 mediates muscle remodeling by cleavage and release of myofibrillar proteins, targeting these proteins for ubiquitination and proteasomal degradation $(34,35)$. The genes $\alpha$-actinin-2-associated LIM protein (ALP), and Leman coiled-coil protein or angiomotin-like protein 2 (LCCP), encoding proteins important for sarcomeric structure (in particular, the $\mathrm{Z}$ line), and Titin immunoglobulin domain protein (TTID), encoding a big, elastic protein that stabilizes sarcomeric filaments (36-38), were additionally upregulated. Another interesting gene displaying increased expression was Popeye protein 3 (POP3). POP genes are expressed in cardiac and skeletal muscles (39), and participate in the synthesis and stabilization of mRNA encoding tubulin (40). Genes encoding ryanodine type 3 ( $\mathrm{RyR} 3$ ), a receptor of the sarcoplasmic reticulum, and calcium homeostasis endoplasmic reticulum protein (CHERP), an intracellular calcium-mobilizing agent (41), were additionally upregulated. 
Table III. Genes encoding sarcomeric proteins.

\begin{tabular}{|c|c|c|c|c|c|}
\hline Subject & Gene ID & $\begin{array}{c}\text { Mean } \\
\log _{2} \mathrm{I}_{\text {post-training }} / I_{\text {pre-training }} \\
\pm \mathrm{SD}\end{array}$ & GB accession & UniGene ID & Gene symbol \\
\hline 2 & Actin, $\alpha 2$, smooth muscle, aorta & $1.29 \pm 0.37$ & NM_001613 & 195851 & ACTA2 \\
\hline 2 & Destrin (actin depolymerizing factor) & $0.83 \pm 0.13$ & NM_006870 & 82306 & DSTN \\
\hline 1 & $\begin{array}{l}\text { Myosin, light polypeptide, regulatory, } \\
\text { non-sarcomeric }(20 \mathrm{kD})\end{array}$ & $0.80 \pm 0.32$ & NM_006471 & 233936 & MLCB \\
\hline 2 & ADP-ribosylation factor-like 5 & $1.09 \pm 0.20$ & NM_012097 & 342849 & ARL5 \\
\hline 2 & Short coiled-coil protein & $1.16 \pm 0.65$ & NM_032547 & 286013 & HRIHFB2072 \\
\hline 2 & $\begin{array}{l}\text { Titin immunoglobulin } \\
\text { domain protein (myotilin) }\end{array}$ & $1.07 \pm 0.26$ & NM_006790 & 84665 & TTID \\
\hline 2 & $\begin{array}{l}\alpha \text {-actinin-2-associated } \\
\text { LIM protein }\end{array}$ & $1.35 \pm 0.70$ & ВC001017 & 135281 & ALP \\
\hline 2 & Leman coiled-coil protein & $1.05 \pm 0.25$ & NM_016201 & 92186 & $\mathrm{LCCP}$ \\
\hline 2 & Ryanodine receptor 3 & $1.40 \pm 0.63$ & NM_001036 & 9349 & RYR3 \\
\hline 1 & $\begin{array}{l}\text { Calcium homeostasis endoplasmic } \\
\text { reticulum protein }\end{array}$ & $1.04 \pm 0.64$ & NM_006387 & 6430 & CHERP \\
\hline 1 & Popeye protein 3 & $0.89 \pm 0.18$ & AK055600 & 303154 & POP3 \\
\hline 2 & Ubiquitin-specific protease 15 & $0.92 \pm 0.18$ & NM_006313 & 23168 & USP15 \\
\hline 5 & $\begin{array}{l}\text { Ubiquitin-conjugating enzyme E2G } 1 \\
\text { (UBC7 homolog, C. elegans) }\end{array}$ & $-2.23 \pm 0.41$ & NM_003342 & 78563 & UBE2G1 \\
\hline 1 & Calpain 3, (p94) & $0.84 \pm 0.22$ & NM_000070 & 40300 & CAPN3 \\
\hline
\end{tabular}

Table IV. Genes involved in oxidative stress.

\begin{tabular}{llcccc}
\hline Subject & \multicolumn{1}{c}{ Gene ID } & \multicolumn{1}{c}{$\begin{array}{c}\text { Mean } \\
\log _{2} I_{\text {post-training }} / I_{\text {post-traning }} \\
\pm \text { SD }\end{array}$} & GB accession & UniGene ID & Gene symbol \\
\hline 2 & Nitric oxide synthase 1 (neuronal) & $-1.18 \pm 0.53$ & NM_000620 & 46752 & NOS1 \\
2 & DNA polymerase epsilon p12 subunit & $-1.14 \pm 0.31$ & NM_019896 & 19980 & P12 \\
2 & Peroxiredoxin 3 & $-1.12 \pm 0.35$ & NM_006793 & 75454 & PRDX3 \\
2 & Thioredoxin & $-1.11 \pm 0.47$ & NM_003329 & 76136 & TXN \\
2 & Methionine sulfoxide reductase A & $-0.93 \pm 0.47$ & NM_014772 & 64096 & MSRA \\
\hline
\end{tabular}

The first column specifies in which of the three different elderly subjects (individuals 1, 2, or 5) the change in expression was detected. The second column lists the common names of the different genes up- or downregulated in the vastus lateralis muscle, when post- and pre-training expression levels were compared. The third column reports changes in expression levels of genes as means of the $\log$ base- 2 of the ratios (log $\mathrm{I}_{\text {post-training }} / \mathrm{I}_{\text {post-training }}$ ), with SD. The fourth column lists Gene Bank Accession numbers, the fifth the UniGene Identification numbers, and the sixth specific gene symbols. Genes involved in energy metabolism were classified as upregulated (positive values) or downregulated (negative values).

Genes involved in oxidative stress. Oxidation of biological substrates, such as DNA, proteins, and lipids, in the elderly, is well-recognized, as oxidants are generated in the mitochondrial respiratory chain, and the deleterious function of such oxidants is particularly important in muscle fibers (1). Vibrational training caused down-regulation of the polymerase $\varepsilon(\mathrm{P} 12)$ gene, encoding a low-affinity enzyme involved in DNA duplication and repair (42) and cell cycle control (Table IV). This enzyme, an important contributor to DNA repair, is involved in the rescue of oxidized DNA, although enzyme activity is less efficient than that of other polymerases. Furthermore, expression of the peroxiredoxin (PRDX3) gene was decreased. PRDX3 is a specific antioxidant enzyme able to remove endogenous cytokine-induced 
$\mathrm{H}_{2} \mathrm{O}_{2}$ (43), using electrons donated by thioredoxin (TXN), the gene for which was also downregulated. PRDX3 encodes a protein that acts as an antioxidant by facilitating the reduction of other proteins via cysteine thioldisulfide exchange. Another gene displaying diminished expression was methionine sulfoxide reductase (MSRA), the protein product of which repairs oxidized methionine (44), one of the most important targets of protein oxidation. Moreover, downregulation of the constitutive nitric oxide synthase (NOS1) was also observed. This gene encodes a constitutive form of the enzyme that synthesizes nitric oxide from L-arginine in the presence of NADPH and $\mathrm{O}_{2}$, and downregulation may be linked to an altered nitric oxide signaling mechanism in aged skeletal muscle (45).

\section{Discussion}

Muscle isometric strength is defined as the maximum force exerted against resistance, and decreases significantly in humans with age (46). Healthy elderly subjects typically display diminished muscle mass, reduced movement velocity and skeletal muscle strength, combining to form the diagnostic characteristics of the condition termed sarcopenia (1).

In recent years, novel 'passive training' methods were proposed for clinical treatment of muscle atrophy and in sport training, consisting of mechanical vibrations applied to specific muscles or over the entire body. A number of investigators showed that such treatments enhance muscle strength and power $(8,47)$, plasma concentrations of testosterone and growth hormone (5), and neuromuscular functions (6). Other studies, however, have drawn opposite conclusions, claiming that no improvement of muscle performance was obtained after vibration treatment (9). In addition, negative effects of whole-body vibration on health were documented. Workers exposed to daily vibration display vascular disorders (10), increased levels of lumbar prolapse, and lower back pain (12).

In this study, the measurements of knee extensor muscle isometric torque, obtained during pre-, during (weeks 4 and 8), and at the end of training, confirmed that local high-intensity vibration increases overall muscle strength in elderly male and female subjects. Specifically, isometric strength was elevated by the fourth week of stimulation, remained high during the training period, and also for at least several weeks after cessation of training. Indeed, follow-up measurements at 16 weeks after the end of training revealed consistently high values of muscle strength, similar to the levels recorded at the end of the vibrational protocol, for both female and male volunteers, although some inter-individual variability was noted.

The sustained increase in lower limb strength suggests modifications in the properties of skeletal muscle. Measurements of thigh circumferences and skinfolds showed that the force increase was not accompanied by an increase in muscle mass. We therefore examined the mechanical properties of single fibers to determine whether the changes can be explained at the cellular level. After 12 weeks of local high-intensity vibration, neither the cross-sectional area nor the specific tension of the $V L$ muscle fibers was changed, compared to pre-training data. Thus, the change in force of the whole extensor muscle group is not based on an alteration in the force-developing ability of single fibers. Previous studies have shown that muscle disuse in the elderly causes a decrease in specific tension (48), whereas resistance training in body builders is associated with increased specific tension (49). Apparently, the improved strength of leg extensor muscles after vibrational training is not related to an increase in muscle fiber-specific tension. Biopsy examination, however, yielded a valuable and unexpected result. The fiber phenotype distribution of the skeletal muscle was altered. The proportion of the fast MyHC-2X isoform was increased, and the proportion of the slow MyHC-1 isoform was significantly lower, in post-training biopsies compared to pre-training biopsies. This shift in myosin isoform expression is a clear indication of changes in fiber type distribution (49). Fiber type transition from slow to $2 \mathrm{X}$, as suggested by the shift in myosin isoforms, should correspond to changes in the proportions of oxidative and glycolytic fibers, because, in human muscles, slow fibers are mainly oxidative whereas $2 \mathrm{X}$ fibers are principally glycolytic (24). Importantly, expression profile analysis validated this metabolic feature. In fact, indications for changes in fiber type distribution were seen in the transcriptional profile. In particular, changes in two of three functional categories (metabolic genes and those encoding sarcomeric proteins) of differentially regulated genes were identified in all screened elderly subjects. Glycolytic and glycogen-dependent metabolism appeared to be stimulated in trained subjects. In this study, personal transcriptional profiles, including data on specific differentially regulated genes, were generated for each of three subjects. Initially, the results appeared inconsistent, but, when the experimental plan was considered, and, particularly, when the long-term nature of the treatment was properly weighted, the transcriptional profiles of the three elderly subjects can be best regarded as specific temporal windows on genes functioning after consolidated or stabilized metabolic (in the wide sense) modifications. We employed a microarray technique to simultaneously screen all pathways activated at a given timepoint, and we were able to detect compensatory pathways activated in the cells. Metabolic genes, either up- or downregulated, were indeed distinct between subjects, but converged into enhancement of the glycogenosynthesis/glycolysis pathway. Glycogenosynthesis is considered as a compensator for increased glucose consumption during training. This hypothesis is consistent with the increased proportion of (specifically) fast MyHC-2X fibers as these fibers employ glycolytic metabolism.

The upregulation of genes, such as actin, destrin, titin and angiomotin, encoding sarcomeric proteins, is consistent with an increase in regeneration that recruits satellite cells to proliferate and fuse into new differentiated myotubes, that are comparable to in vivo primary fibers. We hypothesize that new fibers are generated during the first 4 weeks of local highintensity vibrational stimulation, based on data showing that at week 4, the bilateral isometric strength of lower limbs was significantly increased. We used a biopsy fragment from one subject to collect and grow satellite cells (50), to study whether myogenesis properties in vitro are influenced by local high-intensity vibrational training. We induced the differentiation program in these cells, and, after 7 days of differentiation, we used an antibody MF20 against myosins 
to measure the proportion of myotubes expressing the myosin heavy chain proteins. The preliminary data reveal that, after vibrational training, the proportion of MF20-positive myotubes increased by $11.8 \%$ (from $41.3 \%$ pre-training to $53.1 \%$ posttraining). These data further support the hypothesis that novel fibers stimulated by local high-intensity vibration are induced to differentiate into new, mature, fast MyHC-2X fibers.

Sarcopenic skeletal muscle shows reduced metabolic power because of several factors, such as decreased blood perfusion, increased fibrosis, and development of the atrophic state. Skeletal muscle tissue undergoes constant oxidation, and the main source of reactive oxygen species is the mitochondrion. A previous study by our group showed consistent oxidative damage in aged skeletal muscle tissue (1). Altered metabolism impairs the balance between oxidative stress and scavenger activity. In fact, several antioxidant genes were downregulated in the elderly, suggesting that ROS scavenger activity is insufficient. Peroxiredoxin is an important antioxidant enzyme that counteracts lipid peroxidation, protecting cells by removing $\mathrm{H}_{2} \mathrm{O}_{2}$ (51). Thioredoxin plays a key role in oxidation-reduction reactions (43). Methionine sulfoxide reductase A counteracts methionine oxidation of proteins (44), whereas polymerase $\varepsilon$ is able to repair oxidized DNA, albeit at low efficiency (42). The downregulated constitutive NO synthase (NOS) could be responsible for lowered nitric oxide production and may disrupt nitric oxide signaling in skeletal muscle arterioles, thus impairing vasodilatation in old age (45). Also, in a study of atrophy induction by horizontal bed rest in young males, it was observed that sarcolemmal NOS1 immunofluorescence in the vastus lateralis muscle increased (52). These opposite effects may be explained by age-related variations in muscle conditioning. In fact, during aging, the bioavailability of substrates and cofactors important for NOS activity decreases (45), and this was not reversed by high intensity vibrational training applied to elderly subjects.

Only one elderly subject (subject 2, Table IV) showed downregulation of antioxidant genes mentioned above. This observation suggests that local high-intensity vibration exacerbate existing physiological and personal gene regulation conditions.

Overall, the results of our study indicate that vibrational training was accompanied by several changes at the muscle molecular level. However, the lack of correlation between marked increases in the force developed during maximal voluntary contraction on the one hand, and muscle mass, single fiber thickness, or specific tension, on the other, suggests that nervous control of contraction must also be considered as possibly influenced by our training protocol. Improved recruitment or better activation of motor units might also be invoked to explain the observed increases in muscle force. A recent study by Fattorini et al (14) provided evidence of long-lasting changes in proprioceptive motor control after vibrational training at $100 \mathrm{~Hz}$, and such changes may be partly responsible for the improved contractile performance observed here.

In conclusion, our data validate the effectiveness of the local high-intensity vibrational training procedure to counteract sarcopenia. The technique is easy to use, requires little patient commitment or time, and can be employed on patients with joint and/or neuro-muscular disorders.

\section{Acknowledgements}

We thank Dr Bruno Loffredo for cardiological consultations and Professor Enzo Ballone for assistance with statistical analysis. This study was supported by research grants awarded to Professors R. Saggini, G. Fanò, and S. Fulle from the University 'G. d'Annunzio' of Chieti-Pescara.

\section{References}

1. Fulle S, Protasi F, Di Tano G, et al: The contribution of reactive oxygen species to sarcopenia and muscle ageing. Exp Geront 39: 17-24, 2004.

2. Argiles JM, Busquets S, Felipe A and Lopez-Soriano FJ: Muscle wasting in cancer and ageing: cachexia versus sarcopenia. Adv Gerontol 18: 39-54, 2006.

3. Avila-Funes JA and García-Mayo EJ: The benefits of doing excercise in the elderly. Gac Med Mex 140: 431-436, 2004.

4. Mühlberg W and Sieber C: Sarcopenia and frailty in geriatric patients: implications for training and prevention. Gerontol Geriatr 37: 2-8, 2004.

5. Bosco C, Cardinale M and Tsarpela O: Influence of vibration on mechanical power and electromyogram activity in human arm flexor muscles. Eur J Appl Physiol Occup Physiol 79: 306-311, 1999.

6. Bosco C, Iacovelli M, Tsarpela O, et al: Hormonal responses to whole-body vibration in men. Eur J Appl Physiol 81: 449-454, 2000.

7. Kvorning T, Bagger M, Caserotti P and Madsen K: Effects of vibration and resistance training on neuromuscular and hormonal measures. Eur J Appl Physiol 96: 615-625, 2006.

8. Bosco C, Colli R, Introini E, et al: Adaptive responses of human skeletal muscle to vibration exposure. Clin Physiol 19: 183-187, 1999.

9. De Ruiter CJ, Van Raak SM, Schilperoort JV, Hollander AP and de Hann A: The effects of 11 weeks whole body vibration training on jump height, contractile and activation of human knee extensors. Eur J Appl Physiol 90: 595-600, 2003.

10. Bovenzi M: Exposure-response relationship in the hand-arm vibration syndrome: an overview of current epidemiology research. Int Arch Occup Environ Health 71: 509-519, 1998.

11. Bovenzi M, D'Agostin F, Rui F, Ambrosi L and Zefferino R: Salivary endothelin and vascular disorders in vibration-exposed workers. Scand J Work Environ Health 34: 133-141, 2008.

12. Lings $S$ and Leboeuf-Yde $C$ : Whole-body vibration and low back pain: a systematic, critical review of the epidemiological literature 1992-1999. Int Arch Occup Environ Health 73: 290-297, 2000.

13. Seidel H: On the relationship between whole-body vibration exposure and spinal health risk. Ind Health 43: 361-377, 2005.

14. Fattorini L, Ferraresi A, Rodio A, Azzena GB and Filippi GM: Motor performance changes induced by muscle vibration. Eur $\mathrm{J}$ Appl Physiol 98: 79-87, 2006.

15. Ribot-Ciscar E, Rossi-Durand C and Roll JP: Muscle spindle activity following muscle tendon vibration in man. Neurosci Lett 258: 147-150, 1998 .

16. Eklund $\mathrm{G}$ and Hagbarth KE: Normal variability of tonic vibration reflexes in man. Exp Neurol 16: 80-92, 1966.

17. Brown MC, Enberg I and Matthews PB: The use of vibration as a selective repetitive stimulus for Ia afferent fibers. J Physiol 191: 31-32, 1967.

18. Crone $\mathrm{C}$ and Nielsen J: Central control of disynaptic reciprocal inhibition in humans. Acta Physiol Scand 152: 351-363, 1994.

19. Brunetti O, Filippi GM, Lorenzini M, et al: Improvement of posture stability by vibratory stimulation following anterior cruciate ligament reconstruction. Knee Surg Sports Traumatol Arthrosc 14: 1180-1187, 2006.

20. Bottinelli R, Canepari M, Pellegrino MA, et al: Force-velocity properties of human skeletal muscle fibers: myosin heavy chain isoform and temperature dependence. J Physiol 495: 573-586, 1996.

21. Toniolo L, Maccatrozzo L, Patruno M, et al: Fiber types in canine muscles: myosin isoform expression and functional characterization. Am J Physiol 292: C1915-C1926, 2007.

22. Talmadge RJ and Roy RR: Electrophoretic separation of rat skeletal muscle myosin heavy-chain isoforms. J Appl Physiol 75: 2337-2340, 1993.

23. Jonnalagadda $\mathrm{S}$, and Srinivasan R: Principal components analysis based methodology to identify differentially expressed genes in time-course microarray data. BMC Bioinformatics 9: 267,2008 . 
24. Schiaffino S and Reggiani C: Molecular diversity of myofibrillar proteins: gene regulation and functional significance. Physiol Rev 76: 371-423, 1996.

25. Delibegovic M, Armstrong CG, Dobbie L, Watt PW, Smith AJ and Cohen PT: Disruption of the striated muscle glycogen targeting subunit PPP1R3A of protein phosphatase 1 leads to increased weight gain, fat deposition, and development of insulin resistance. Diabetes 52: 596-604, 2003.

26. Schiaffino S, Sandri, M and Murgia M: Activity-dependent signaling pathways controlling muscle diversity and plasticity. Physiology 22: 269-278, 2007.

27. Smolle M, Prior AE, Brown AE, Cooper A, Byron O and Lindsay JG: A new level of architectural complexity in the human pyruvate dehydrogenase complex. J Biol Chem 281: 19772-19780, 2006.

28. Bishop D, Edge J, Thomas C and Mercier J: High-intensity exercise acutely decreases the membrane content of MCT1 and MCT4 and buffer capacity in human skeletal muscle. J Appl Physiol 102: 616-621, 2007.

29. Abe T, Kakyo M, Tokui T, et al: Identification of a novel gene family encoding human liver-specific organic anion transporter LST-1. J Biol Chem 274: 17159-17163, 1999.

30. Durand E, Boutin P, Meyre D, Charles MA, Clement K, Dina C and Froguel P: Polymorphisms in the amino acid transporter solute carrier family 6 (neurotransmitter transporter) member 14 gene contribute to polygenic obesity in French Caucasians. Diabetes 53: 2483-2486, 2004

31. Hu WH, Hausmann ON, Yan MS, Walters WM, Wong PK and Bethea JR: Identification and characterization of a novel Nogointeracting mitochondrial protein. J Neurochem 81: 36-45, 2002.

32. Estornes Y, Gay F, Gevrey JC, Navoizat S, et al: Differential involvement of destrin and cofilin-1 in the control of invasive properties of Isreco1 human colon cancer cells. Int J Cancer 121: 2162-2171, 2007.

33. Panic B, Whyte JR and Munro S: The ARF-like GTPases Arl1p and Arl3p act in a pathway that interacts with vesicle-tethering factors at the Golgi apparatus. Curr Biol 13: 405-410, 2003.

34. Sorimachi H, Toyama-Sorimachi N, Saido TC, et al: Musclespecific calpain, p94, is degraded by autolysis immediately after translation, resulting in disappearance from muscle. J Biol Chem 268: 10593-10605, 1993.

35. Kramerova I, Kudryashova E, Venkatraman G and Spencer MJ: Calpain 3 participates in sarcomere remodeling by acting upstream of the ubiquitin-proteasome pathway. Hum Mol Genet 14: 2125-2134, 2005.

36. Tskhovrebova L and Trinick J: Titin: properties and family relationships. Nat Rev Mol Cell Biol 4: 679-689, 2003.

37. Labeit S, Lahmers S, Burkart C, et al: Expression of distinct classes of titin isoforms in striated and smooth muscles by alternative splicing, and their conserved interaction with filamins. J Mol Biol 362: 664-681, 2006

38. Bratt A, Wilson WJ, Troyanovsky B, Aase K, Kessler R, Van Meir EG and Holmgren L: Angiomotin belongs to a novel protein family with conserved coiled-coil and PDZ binding domains. Gene 298: 69-77, 2002.
39. Andrée B, Hillemann T, Kessler-Icekson G, Schmitt-John T, Jockusch $\mathrm{H}$, Arnold $\mathrm{HH}$ and Brand T: Isolation and characterization of the novel popeye gene family expressed in skeletal muscle and heart. Dev Biol 223: 371-382, 2000.

40. Ochotorena IL, Hirata D, Kominami K, et al: Conserved Wat1/Pop3 WD-repeat protein of fission yeast secures genome stability through microtubule integrity and may be involved in mRNA maturation. J Cell Sci 114: 2911-2920, 2001.

41. Laplante JM, O'Rourke F, Lu X, Fein A, Olsen A, and Feinstein MB: Cloning of human $\mathrm{Ca}^{2+}$ homoeostasis endoplasmic reticulum protein (CHERP): regulated expression of antisense cDNA depletes CHERP, inhibits intracellular $\mathrm{Ca}^{2+}$ mobilization and decreases cell proliferation. Biochem J 348: 189-199, 2000.

42. Sugino A, Ohara T, Sebastian J, Nakashima N and Araki H: DNA polymerase epsilon encoded by cdc $20^{+}$is required for chromosomal DNA replication in the fission yeast schizosaccharomices pombe. Genes Cells 3: 99-110, 1998.

43. Nordberg J and Arnér ES: Reactive oxygen species, antioxidants, and the mammalian thioredoxin system. Free Radic Biol Med 31: $1287-1312,2001$

44. Vattanaviboon P, Seeanukun C, Whangsuk W, Utamapongchai S and Mongkolsuk S: Important role for methionine sulfoxide reductase in the oxidative stress response of Xanthomonas campestris pv. Phaseoli J Bacteriol 187: 5831-5836, 2005.

45. Delp MD, Behnke BJ, Spier SA, Wu G and Muller-Delp JM: Ageing diminishes endothelium-dependent vasodilatation and tetrahydrobiopterin content in rat skeletal muscle arterioles. J Physiol 586: 1161-1168, 2008.

46. Doherty TJ: Invited review: Aging and sarcopenia. J Appl Physiol 95: 1717-1727, 2003.

47. Samuelson B, Jorfeldt L, Ahlborg B: Influence of vibration on endurance of maximal isometric contraction. Clin Physiol 9: 21-25, 1989.

48. D'Antona G, Pellegrino MA, Adami R, et al: The effect of ageing and immobilization on structure and function of human skeletal muscle fibers. J Physiol 552: 499-511, 2003.

49. D'Antona G, Lanfranconi F, Pellegrino MA, et al: Skeletal muscle hypertrophy and structure and function of skeletal muscle fibers in male body builders. J Physiol 570: 611-627, 2006.

50. Beccafico S, Puglielli C, Pietrangelo T, Bellomo R, Fanò G and Fulle S: Age-dependent effects on functional aspects in human satellite cells. Ann NY Acad Sci 1100: 345-352, 2007.

51. Rhee SG, Chae HZ and Kim K: Peroxiredoxins: a historical overview and speculative preview of novel mechanisms and emerging concepts in cell signalling. Free Radic Biol Med 38: $1543-1552,2005$

52. Rudnick J, Püttmann B, Tesch PA, et al: Differential expression of nitric oxide synthases (NOS 1-3) in human skeletal muscle following exercise countermeasure during 12 weeks of bed rest. FASEB J 18: 1228-1230, 2004. 